\title{
On The Closed Geodesics Problem
}

\author{
Francesco Mercuri
}

\section{Paolo Piccione}

\begin{abstract}
In this paper we review some important results on the closed geodesics problem for compact Riemannian manifolds, as the Gromoll-Mayer Theorem, and discuss some extension of those results to the case of Finsler and semi Rimannian manifolds.
\end{abstract}

\section{Introduction.}

The study of closed geodesics in a Riemannian manifold is a classical theme of research in Riemannian geometry. A basic question is:

The closed geodesic problem: Find a (sharp) lower bound for the number of non constant geometrically distinct ${ }^{1}$ closed geodesics on a compact manifold $M$.

In this paper we will describe some classical results in this direction, leading to the following:

Conjecture: On any compact Riemannian manifold there are infinitlely many closed geodesics.

We will discuss also some partial generalizations and related open questions for the the corresponding problem for Finsler and semi-Riemannian manifolds.

The starting point in attacking the problem is the way we look at a closed geodesic. In fact a closed geodesic can be viewed in two different ways:

- as a closed curve which is a geodesic,

Key words: Riemannian manifolds, Finsler manifolds, semi Riemannian manifolds, closed geodesics, Morse inequalities.

Work partially supported by FAPESP and CNPq, Brazil.

${ }^{1}$ Two closed geodesics are geometrically distinct if one is not obtained from the other by a rotation or an iteration. 
- as a geodesic which is a closed curve.

If we take the first point of view, our "universe" is the space of closed curves and we try to find the ones that are geodesics; this is the variational viewpoint. For the second point of view the "universe" is the space of geodesics and we try to find the ones that are closed; this is the dynamical viewpoint.

In order to determine closed curves that are geodesics, the standard technique is critical point theory. The geodesics are the critical points of the energy functional, and we want to estimate the number of critical points of the functional in terms of topological invariants of the manifold.

In the second case we look at the geodesic flow in the unitary tangent bundle. If there is a non constant closed orbit of the flow ( see Theorem 2.5), we look at the return map, i.e. we look at an orthogonal section at a point, call it $\Sigma$, and, for points of $\Sigma$ near by the given one, to the orbit starting at this point. This orbit will meet again $\Sigma$ in a return point. We look at the periodic points of this map, prove that are generically infinitely many, and that this "generically" can be intended in the sense of the metrics. This is, essentially, the point of view of dynamical systems.

The main results on the problem are the following:

Theorem 1.1. (Gromoll-Meyer) Let $M$ be an n-dimensional simply connected Riemannian manifold whose topology is sufficiently complicated. Then there are infinitely many non trivial, geometrically distinct closed geodesics.

Remark 1.2. The terms sufficiently complicated will be cleared in the precise statement of the the Theorem given in section 2 (see Theorem 2.12). It is also interesting to observe that Gromoll-Meyer's result holds more generally for a class of non simply connected manifolds (see Example at the end of Section 4).

Theorem 1.3. (Klingenberg-Takens) Let $M$ be a compact manifold. There is a dense set of metrics on $M$ such that every metric in this set admits infinitely many non trivial, geometrically distinct closed geodesics.

In this paper we will be essentially interested in understanding Theorem 1.1 and some generalizations. In next section we will describe the basic critical point theory for proving Theorem 1.1, and, in the following ones, we will discuss some generalizations of the result to the case of Finsler and semi-Riemannian metrics. 


\section{The critical point theory.}

We will start this section discussing the basic critical point theory. We will consider a connected Riemannian manifold $\mathcal{M}$ modeled on an Hilbert space $\mathbb{H}$ and a smooth map $f: \mathcal{M} \longrightarrow \mathbb{R}$. We recall that a point $x \in \mathcal{M}$ is critical if the differential $\mathrm{d} f(x): T_{x} \mathcal{M} \longrightarrow \mathbb{R}$ is the zero map.

We will assume the following:

- $\mathcal{M}$ is a complete metric space ${ }^{2}$.

- $f$ is smooth and bounded below.

- The following compacness condition is fullfilled:

If $\left\{x_{n}\right\} \subseteq \mathcal{M}$ is a sequence such that $\left|f\left(x_{n}\right)\right|$ is bounded and inf $\left\|\nabla f\left(\bar{x}_{n}\right)\right\|=0$, then $\left\{x_{n}\right\}$ has a convergent subsequence.

Remark 2.1. The last condition is known as Condition $(C)$ or Palais-Smale condition. It allows to carry on compactness arguments, even in the infinite dimensional case.

In the situation above we have the Theorem of Lusternik-Schnirelman:

Theorem 2.2. (Lusternik-Schnirelman) In the hypothesis above, $f$ has at least ${ }^{3}$ cat $(\mathcal{M})$ critical points.

Remark 2.3. For further use we we recall that the idea of the proof of Theorem 2.2 is to study the flow of $-\nabla f$, where $\nabla f$ is the gradient of $f$. Set $f^{c}=\{x \in \mathcal{M}: f(x) \leq c\}$. If there are no critical values in $[a, b] \subseteq \mathbb{R}$, following the integral lines of $-\nabla f$, we can deform $f^{b}$ onto $f^{a}$, so there is no change in the topology, passing from $f^{b}$ to $f^{a}$. If $c$ is the only critical value in $[a, b]$, the category of $f^{b}$ at least the one of $f^{a}$ plus the category of the set of critical points in $f^{-1}(c)$.

For a detailed proof see [20], [21].

To apply such a Theorem to the closed geodesics problem we proceed as follows. Let $M$ be a compact Riemannian manifold. Consider the space:

$$
\tilde{\Lambda}(M):=\left\{\gamma: S^{1} \longrightarrow M: \gamma \in C^{\infty}\left(S^{1}, M\right)\right\},
$$

\footnotetext{
${ }^{2}$ With respect to the metric:

$d(x, y)=\inf \left\{\int_{0}^{1}\|\dot{\gamma}(t)\| \mathrm{d} t: \gamma:[0,1] \longrightarrow \mathcal{M}\right.$ is a smooth curve with $\left.\gamma(0)=x, \gamma(1)=y\right\}$.

${ }^{3}$ We recall that, for a topological spaces $Y \subseteq X, \operatorname{cat}(Y, X)$ is defined as the minimal number (possible infinity) of closed sets in $X$, contractible in $X$, which cover $Y$. Then $\operatorname{cat}(X):=\operatorname{cat}(X, X)$.
} 
and the energy functional:

$$
E: \tilde{\Lambda}(M) \longrightarrow \mathbb{R}, \quad E(\gamma):=\frac{1}{2} \int_{S^{1}}\|\dot{\gamma}(t)\|^{2} \mathrm{~d} t
$$

The "critical points" of the energy functional are the closed geodesics. Unfortunately $\tilde{\Lambda}(M)$ does not have any natural structure of complete Riemannian manifold. We will then use the following strategy:

- We define a suitable complete Riemannian manifold $\Lambda(M)$ which contains $\tilde{\Lambda}(M)$ as a dense subset.

- We extend the energy functional to a functional on $\Lambda(M)$ which is smooth and bounded below.

- We prove that the extended functional verify condition $(C)$.

- We prove that the critical points of the extended functional are in $\tilde{\Lambda}(M)$ (regularity Theorem).

Remark 2.4. The choice of the completion of $\tilde{\Lambda}(M)$ is, in our case, very simple and natural (see below). However, for general variational problems, this task is quite delicate. In fact we have to take in account two "conflicting interests": On one hand we want a topology in which convergence is easy to show, which will help in proving condition $(C)$, and on the other hand we want a topology not too different from the natural $C^{\infty}$ topology, in order to prove the regularity Theorem.

We will assume that $M$ is a submanifold of $\mathbb{R}^{N}$, with the induced metric, which is not restrictive by the Nash embedding Theorem. Nevertheless, it is interesting to observe that a critical point theory setup for the closed geodesic problem can be constructed in abstract Riemannian manifolds, without using any specific isometric embedding into Euclidean spaces. This abstract setup is particularly useful when dealing with complete but non compact manifold; note in fact that in this case Nash embedding theorem does not guarantee the existence of a closed embedding.

Consider the vector space of maps $\gamma: S^{1} \longrightarrow \mathbb{R}^{N}$ such that:

(1) $\dot{\gamma}(t)$ exist for almost all $t \in S^{1}$, and $\|\dot{\gamma}(t)\| \in L^{2}\left(S^{1}, \mathbb{R}^{N}\right) \subseteq L^{1}\left(S^{1}, \mathbb{R}^{N}\right)$,

(2) $\gamma(t)=\gamma(0)+\int_{S^{1}} \dot{\gamma}(t) \bar{d} t$, i.e., $\gamma$ is absolutely continuous.

and denote this space by $H^{1}\left(S^{1}, \mathbb{R}^{N}\right)$. This space is an Hilbert space with respect to the scalar product:

$$
(\gamma, \tau):=\langle\gamma(0), \tau(0)\rangle+\int_{S^{1}}\langle\dot{\gamma}(t), \dot{\tau}(t)\rangle \mathrm{d} t
$$


and the set:

$$
\Lambda(M):=\left\{\gamma \in H^{1}\left(S^{1}, \mathbb{R}^{N}\right): \gamma(t) \in M, \forall t \in S^{1}\right\},
$$

is a closed (hence complete) submanifold of $H^{1}\left(S^{1}, \mathbb{R}^{N}\right)$.

The tangent space of $\Lambda(M)$ at $\gamma$ is the space:

$$
T_{\gamma} \Lambda(M)=\left\{\xi \in H^{1}\left(S^{1}, \mathbb{R}^{N}\right): \xi(t) \in T_{\gamma(t)} M\right\},
$$

and the Riemannian metric is given by:

$$
(\xi, \eta):=\int_{S^{1}}\left[\left\langle\xi^{\prime}, \eta^{\prime}\right\rangle+\langle\xi, \eta\rangle\right] \mathrm{d} t,
$$

where primes denote covariant derivative along $\gamma$ and $\langle\cdot, \cdot\rangle$ is the Riemannian metric of $M$.

Then we have:

- $\Lambda(M)$ is a complete Riemannian manifold.

- The energy functional extend naturally to a map $E: \Lambda(M) \longrightarrow \mathbb{R}$ which is smooth and bounded below (obvious).

- $E: \Lambda(M) \longrightarrow \mathbb{R}$ verify condition $(C)$ (relatively easy, see [21] for example).

- The critical points of $E$ are closed geodesics (easy, see [21] for example).

There are various problems if we want to apply Theorem 2.2 to our situation in a naive way.

- We already know that there exist infinitely many closed geodesics, the constant ones! We can avoid this problem considering a "relative" version of 2.2. Identifying $M$ with the constant curves, the number of non constant closed geodesics can be estimate in terms of the topology of the pair $(\Lambda(M), M)$. In fact, in this way we can prove:

Theorem 2.5. On a compact Riemannian manifold the is at least one non constant closed geodesic.

- If we start with a nontrivial closed geodesic $\gamma: S^{1} \longrightarrow M$, we can produce infinitely many other closed geodesics, simply "rotating" $\gamma$. We can avoid this problem with an equivariant version of Theorem 2.2. We consider the action:

$$
\mu: S^{1} \times \Lambda(M) \longrightarrow \Lambda(M), \quad \mu(\theta, \gamma)(t)=\gamma(t+\theta),
$$

where $\theta, t \in \mathbb{R} / \mathbb{Z}$. The energy functional is invariant by this action and so induces a functional defined in the quotient space $\Pi(M)=\Lambda(M) / \mu$. 
Although $\Pi(M)$ is not a manifold ${ }^{4}$, we can still prove a version of Theorem 2.2 that we can apply to this situation.

But the real problem in this approach is the following: how we can distinguish a given closed geodesic $\gamma: S^{1} \longrightarrow M$ from its iterates $\gamma_{n}(t)=$ $\gamma(n t)$ ? Theorem 2.2 and its simpler generalizations, like the ones quoted above, are not fine enough to answer the question. So we need finer results that we will describe now. We recall the following concepts:

- The Hessian map $H(f, x)$ is defined as:

$$
H(f, x): T_{x} \mathcal{M} \times T_{x} \mathcal{M} \longrightarrow \mathbb{R}, \quad H(f, x)(X, Y):=\tilde{X} \tilde{Y}(f)(x),
$$

where $\tilde{X}, \tilde{Y}$ are extensions of $X, Y$ to a neighborhood of $x$. If $x$ is a critical point of $f, H(f, x)(X, Y)$ does not depend on the choice of the extensions $\tilde{X}, \tilde{Y}$, and is a well defined symmetric bilinear form.

- A critical point of $f$ is non degenerate if the Hessian map is non degenerate.

- The index of $f$ at a critical point is the supremum of the dimensions of subspaces on which the Hessian is negative definite (eventually infinite).

The behavior of a function near a non degenerate critical point is described by the classical Morse Lemma:

Lemma 2.6. (Morse Lemma) Let $\mathbb{H}$ be an Hilbert space, $U$ an open neighborhood of $0 \in \mathbb{H}$ and $f: U \longrightarrow \mathbb{R}$ a $C^{2}$ function such that $f(0)=0$ and $0 \in U$ is a non degenerate critical point. Then there exist an orthogonal decomposition

$$
\mathbb{H}=\mathbb{H}_{-} \oplus \mathbb{H}_{+},
$$

such that $\mathbb{H}_{ \pm}$are invariant under the Hessian of $f$ at 0 , and, up to a local diffeomorphism of a neigborhood $V$ of $0 \in V \subseteq U$, we have:

$$
f(x)=-\left\|x_{-}\right\|^{2}+\left\|x_{+}\right\|^{2}, \quad x \in V,
$$

where $x_{ \pm}$are the orthogonal projections of $x$ onto $\mathbb{H}_{ \pm}$.

Observe that, in particular, the non degenerate critical points are isolated.

Let $p \in \mathcal{M}$ be a non degenerate critical point of index $\lambda$, with $f(p)=c$ and suppose, for simplicity, that $p$ is the only critical point in $f^{-1}([a, b])$, $a<c<b$. A simple excision argument, the Morse Lemma 2.6 and deformation along the integral lines of $-\nabla f$ give:

\footnotetext{
${ }^{4}$ In fact there are two main problems: The action of $S^{1}$ is not smooth, and the non constant curves have, in general, non conjugate isotropy groups.
} 
Proposition 2.7. Let $A$ be a commutative ring with unity. Then:

$$
H_{k}\left(f^{b}, f^{a} ; A\right) \cong A \text {, if } k=\lambda, H_{k}\left(f^{b}, f^{a}\right)=\{0\} \text {, if } k \neq \lambda,
$$

where $H_{k}\left(f^{b}, f^{a} ; A\right)$ is the relative $k$-dimensional singular homology with coefficients in $A$.

As a consequence we have:

Theorem 2.8. (Morse inequalities)

$$
c_{\lambda} \geq b_{\lambda}(\mathcal{M} ; \mathbb{F})
$$

where $c_{\lambda}$ is the number of critical points of index $\lambda<\infty, \mathbb{F}$ is a field, and $b_{\lambda}(\mathcal{M} ; \mathbb{F})$ is the $\lambda^{\text {th }}$ Betti number of $\mathcal{M}$ with coefficients in $\mathbb{F}$, i.e. the dimension, over $\mathbb{F}$, of $H_{\lambda}(\mathcal{M} ; \mathbb{F})$, the $\lambda^{\text {th }}$ homology group of $\mathcal{M}$ with coefficients in $\mathbb{F}$.

Remark 2.9. Observe that Theorem 2.8 gives no information as to the existence of critical points of infinite index, in terms of the singular homology of $\mathcal{M}$. In the last few years there has been interest in finding other "homological invariants" that can give extimates on the existence of such critical points (see [1], for example).

Although Theorem 2.8 gives an idea how to proceed in the proof of Theorem 1.1, it is not yet satisfactory, since a closed geodesic $\gamma$ cannot be a non degenerate critical point of the energy. In fact it is not an isolated critical point since the entire orbit $S^{1} \gamma$, which is diffeomorphic to $S^{1}$ if $\gamma$ is not constant, is a set of critical points. For dealing with this problem we consider the analogue of Theorem 2.8 for non degenerate critical submanifolds.

Definition 2.10. A connected submanifold $N \subseteq \mathcal{M}$ is a non degenerate critical submanifold if all point in $N$ are critical points of $f$ and for each $x \in N$ the restriction of the hessian of $f$ to the normal space at $x$, is non degenerate. The index of such a submanifold is the index of the hessian form restricted to the normal space.

It turns out that, in this case, the nullity is the dimension of $N$ and the index is well defined, i.e., it is constant along $N$.

We can considere a small tubular neighborhood of $N$, for example, if $N$ is compact, the exponential of an $\epsilon$ neighborhood of the zero section of the normal bundle, and apply 2.7 to the restriction of $f$ to the fibres. Collecting the local results, we get:

Theorem 2.11. If $N_{i}, i=1, \ldots l$ are the non degenerate compact critical submanifolds in $f^{-1}([a, b])$ and $\lambda_{i}$ is the index of $N_{i}$, we have:

$$
H_{*}\left(f^{b}, f^{a}\right) \cong \oplus H_{*-\lambda_{i}}\left(M_{i}\right) .
$$


For a detailed proof, see, for example, [4].

Now we state precisely Theorem 1.1 and give an idea of the proof.

Theorem 2.12. Let $M$ be a compact simply connected riemannian manifold such that the sequence of Betti numbers $\beta_{i}(\Lambda(M))$ is unbounded (with some field of coefficients). Then $M$ admits infinitely many prime ${ }^{5}$, non constant, closed geodesics.

We start observing that hypothesis that $M$ is simply connected imply that $\Lambda(M)$ is connected. Moreover compactness imply that the homology of $\Lambda(M)$ is finitely generated in any dimension, so the Betti numbers are finite. It is interesting to observe that the simple connectedness assumption is not really necessary in Theorem 2.12 , whose main result holds also for a class of non simply connected manifolds. What is really needed in the proof of Theorem 2.12 is that

$$
\limsup _{i \rightarrow \infty} \beta_{i}(\Lambda M)=+\infty
$$

examples of compact manifolds $M$ that are not simply connected, whose free loop space has some of its Betti number infinite, and that satisfies (1) can be found at the end of Section 4 .

We also recall the expression of the index form:

$$
H(E, \gamma)(\xi, \eta)=\int_{0}^{1}\left[\left\langle\xi^{\prime}, \eta^{\prime}\right\rangle-\langle R(\xi, \dot{\gamma}) \dot{\gamma}, \eta\rangle\right] \mathrm{d} t
$$

The constant geodesics give contribution to the homology of $\Lambda(M)$ only in dimension up to $n=\operatorname{dim}(M)$.

Suppose there are a finite number of prime, non constant closed geodesics, $\gamma_{j}, j=1, \ldots k$ and suppose for the moment that the orbits of the $\gamma_{j}^{\prime} s$ and of their iterates are non degenerate critical submanifolds (diffeomorphic to $\left.S^{1}\right)$. By the Morse index Theorem, the index of the $\gamma_{j}^{\prime} s$ is finite. The basic step is to estimate the index of the iterates $\gamma_{j}^{m}$. This was done by Bott (see [5]) and a simpler version appears in [3]:

Lemma 2.13. The index of $\gamma_{j}^{m}$ is either constant (equal to 0), or it has a uniform linear growth in $m$ as $m \rightarrow \infty$.

The Lemma imply that the number of closed geodesics with a given index is uniformly bounded and, by Theorem 2.11, so are the Betti numbers, a contradiction.

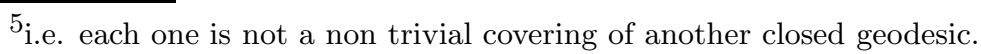


For the possibly degenerate case we need a Morse Lemma that apply in this situation. Let $\mathbb{H}$ be an Hilbert space and $f: U \longrightarrow \mathbb{R}$ a smooth function defined in a neighborhood of $0 \in \mathbb{H}$, with $f(0)=0$. Suppose that 0 is an isolated critical point of index $\lambda$ and finite nullity $\nu$. Them we have a splitting $\mathbb{H}=\mathbb{H}_{ \pm} \oplus \mathbb{H}_{0}$, where $\mathbb{H}_{ \pm}$are as in 2.6, and $\mathbb{H}_{0}$ is the kernel of the symmetric endomorphism determined by the hessian form $\left(\operatorname{dim} \mathbb{H}_{0}=\nu\right)$. If $x \in \mathbb{H}, x=x_{+}+x_{-}+y, x_{ \pm} \in \mathbb{H}_{ \pm}, y \in \mathbb{H}_{0}$. The degenerate Morse Lemma states that, up to a change of variables, the function takes the form:

$$
f(x)=-\left\|x_{-}\right\|^{2}+\left\|x_{+}\right\|^{2}+g(y),
$$

for some function $g$, defined in a neighborhood of 0 , depending only on the $y$ variable. A small perturbation of $g$ with support in a small neighborhood of $0 \in \mathbb{H}_{0}$, will produce a new function with only non degenerate critical points. The indexes of those critical points are between $\lambda$ and $\lambda+\nu$. So, the change in homology passing from $f^{-\epsilon}$ to $f^{\epsilon}$ occurs only in dimension between $\lambda$ and $\lambda+\nu$.

For the case of critical submanifolds we procede as before looking at a small tubular neighborhood of the manifold and working fibrewise.

To proceed as in the non degenerate case we need only to control the nullity of an iterated geodesic in terms of the nullity of the prime one.

For details of the proof we refer to [6] and [7]

Remark 2.14. The condition on the growth of the Betti numbers in Theorem 1.1 may be interpreted in terms of the topology of $M$ as follows: In the hypothesis of the Theorem, taking homology with rational coefficients, the condition is satisfied if and only if the rational cohomology ring of $M$ is not a truncated polynomial ring in one generator. In particular if the cohomology is not isomorphic to the cohomology of a symmetric space of rank one (see [23]).

\section{The Finsler case}

In 1973, Katok give an example of a Finsler metric on $S^{2}$ with only two prime non constant closed geodesics (see [9]). Later Ziller constructed Finsler metrics on manifolds diffeomorphic to compact symmetric spaces of rank one having only finitely many closed geodesics (see [24]). Therefore a Theorem analogue to 1.1, in the context of Finsler geometry is the best possible. In this section we will discuss briefly the closed geodesic problem for Finsler manifolds.

We recall some basic facts:

Definition 3.1. A Finsler metric on a smooth manifold $M$ is a continuous function $F: T M \longrightarrow \mathbb{R}$ such that: 
(1) $F$ is $C^{\infty}$ outside the zero section,

(2) $F(X) \geq 0$ and $F(X)=0$ if and only if $X=0$,

(3) $F(t X)=t F(X) \quad \forall t \in \mathbb{R}, t \geq 0$.

(4) Let $L=F^{2}$. Then the fibre derivative $\mathrm{d}_{f} L: T M \longrightarrow T^{*} M$ is regular outside the zero section.

It is easy to see that $L$ is actually $C^{1}$ on the entire tangent bundle $T M$. Remark 3.2. A Riemannian metric is a Finsler metric, setting $L(X):=$ $\langle X, X\rangle$. In this case $L$ is $C^{\infty}$ on the all $T M$. The low differentiability at the zero section of a Finsler metric caracterizes the ones that do not came from Riemannian metirc. More precisely, the function $L$ is $C^{2}$ on the all $T M$ if and only is the Finsler metric associated to a Riemannian metric.

For a Finsler metric there is not a unique canonical connection. We can use the canonical symplectic structure in the cotangent bundle $T^{*} M$ and its pull back via $\mathrm{d}_{f} L$. This is, by condition (4), a symplectic structure in $T M$, and we can define geodesics using this structure (see [14] and [24], for example). For what we are concerned, we can assume that geodesics are critical points of the energy functional:

$$
E(\gamma):=\frac{1}{2} \int_{S^{1}} L(\dot{\gamma}(t)) \mathrm{d} t .
$$

The analogue of Theorem 1.1, in the context of Finsler manifolds, was first proved by Matthias (see [13]). The author uses a finite dimensional approximation of the space of closed curves, much like Milnor uses in [16] for the fixed endpoints problem.

We can also proceed along the line described in the previous section.

We consider a fixed Riemannian metric on $M$ and the associated manifold $\Lambda(M)$, as in section 1 . We want to develop the Morse Theory for the Finsler energy functional. To proceed as in the Riemannian case we have to work out essentially three points:

- The energy functional in not $C^{2}$, since $L$ is not $C^{2}$ on the zero section of $T M$ (see Remark 3.2). However it is twice differentiable at a closed geodesic, since the tangent vector of such a curve is nowhere zero. The gradient field is locally Lipschitzian, so it's flow is well defined. The part of the Theory concerning deformation along the integral lines of the gradient is, therefore, the same as in the Riemannian case. So we need to prove a Morse Lemma for functions in our differentiability conditions. This has been done in [15] for the non degenerate case and in [17] in the possibly degenerate case. 
- Prove condition $(C)$ for the energy functional and the "regularity Theorem", i.e. that the critical points are actually smooth. This was done in [14].

- We need an estimate for the index and nullity of an iterated closed geodesic. This was done in [13] and [22].

With those two results we conclude, as in the Riemannian case:

Theorem 3.3. Let $M$ be a compact simply connected Finsler manifold such that the sequence of Betti numbers of $\Lambda(M)$ is not bounded. Then there are infinitely many prime closed geodesics.

We conclude this section by presenting a curious result on the existence of infinitely many closed geodesic, in the Finsler case, from the point of view of Hamiltonian systems.

Theorem 3.4. Let $M$ be a compact differentiable manifold. Then, in the set of $C^{2}$ Finsler metrics, there is a dense subset such that for each metric in this subset, the set of unit vectors whose associated geodesic is closed, is dense in the $C^{2}$ topology.

Remark 3.5. Theorem 3.4 is proved in [24]. It follows from an analogous result for general Hamiltonian systems, where the unit tangent bundle is replaced by a constant energy surface. It is interesting to observe that Theorem 3.4 does not hold in the space of Riemannian metrics ${ }^{6}$, not even in the space of symmetric Finsler metrics ${ }^{7}$. Theorem 1.3 is a much weaker result.

\section{The semi-Riemannian case}

There are several obstructions to Morse theory in the case of geodesics in manifolds endowed with a non positive definite metric tensor $g$ (semiRiemannnian manifolds. First, the geodesic action functional:

$$
E: \Lambda(M) \longrightarrow \mathbb{R}, \quad E(\gamma)=\frac{1}{2} \int_{S^{1}} g(\dot{\gamma}, \dot{\gamma}) \mathrm{d} t
$$

is not bounded from below and it does not satisfy condition (C); besides, the Morse index of each critical point is infinite. Let us consider the case of stationary Lorentzian manifolds (i.e., manifolds endowed with a metric tensor of index 1), that admit a complete timelike Killing vector field $Y$. Assume also that $M$ has a compact Cauchy surface; recall that a Cauchy surface in a Lorentzian manifold is a spacelike hypersurface $S$ with the property that every inextendible causal curve intercepts $S$ exactly once. Recall that

\footnotetext{
${ }^{6}$ The set of Riemannian metrics is not open in the space of Finsler metrics.

${ }^{7}$ A Finsler metric $F$ is symmetric if $F(-X)=F(X)$.
} 
all Cauchy surfaces of a Lorentzian manifold (assuming the existence of at least one) are homeomorphic; for the background in Lorentzian geometry we refer to the textbooks [2] and [18]. Lorentzian manifolds admitting a Cauchy surface are said to be globally hyperbolic. Timelike invariance of the metric tensor allows to determine a smooth embedded submanifold $\mathcal{N}$ of $\Lambda M$ with the following properties:

- $\left.E\right|_{\mathcal{N}}$ has the same critical points of $E$;

- $\mathcal{N}$ has the same homotopy type of $\Lambda(M)$ (this uses the completeness of the timelike Killing vector field);

- $E$ is bounded from below and it satisfies condition (C) on each connected component of $\mathcal{N}$ (this uses the compactnes of the Cauchy surface);

- each critical point of $\left.E\right|_{\mathcal{N}}$ has finite Morse index;

- if a critical point is degenerate for $\left.E\right|_{\mathcal{N}}$, then it is also degenerate for $E$.

More precisely, $\mathcal{N}$ is the set of all closed curves $\gamma$ in $M$, having Sobolev regularity $H^{1}$, for which the quantity $g(\dot{\gamma}, Y)$ is constant almost everywhere.

The abelian group $G=\mathrm{O}(2) \times \mathbb{R}$ acts (isometrically) on $\mathcal{N}$, and $E$ is $G$ invariant. The group $\mathrm{O}(2)$ acts on the parameter space $S^{1}$ of the curves, and as in the Riemannian case, this action is not smooth, but only continuous. Nevertheless, if $\gamma$ is a smooth curve, then the orbit $\mathrm{O}(2) \gamma$ is smooth, and it is diffeomorphic to $\mathrm{O}(2)$ if $\gamma$ is not constant. In particular, critical orbits are always smooth. The group $\mathbb{R}$ acts by translation along the flow lines of the timelike Killing vector field $Y$; obviously, the actions of $\mathrm{O}(2)$ and of $\mathbb{R}$ commute. In this situation, we define geometrically distinct two closed geodesics that belong to different $G$-orbits, and that cannot be obtained one from another by iteration. The action of $\mathbb{R}$ is free, the orbit space given by the quotient $\tilde{\mathcal{N}}=\mathcal{N} / \mathbb{R}$ is a smooth manifold and $\mathcal{N}$ is diffeomorphic to the product $\widetilde{\mathcal{N}} \times \mathbb{R}$. Thus, in order to study multiplicity of distinct closed geodesics, it suffices to study geometrically distinct critical $\mathrm{O}(2)$-orbits for the constrained functional $\left.E\right|_{\widetilde{\mathcal{N}}}$.

The central result of [3], which gives the existence of infinitely many distinct closed geodesics in a class of stationary Lorentzian manifolds, is obtained applying equivariant Morse theory to this setup. Essential tools for the development of the theory are a calculation of the Morse index for each critical point of $\left.E\right|_{\mathcal{N}}$, and a formula that describes its growth under iterations. The Morse index is given in terms of symplectic invariants of the geodesic, such as the Conley-Zehnder and the Maslov index. Using a method for computing the index of essentially positive symmetric bilinear forms, possibly degenerate, in terms of restrictions to possibly degenerate subspaces, one reduces the computation of the Morse index for periodic 
geodesics to the Morse index of the corresponding fixed endpoint geodesic, avoiding the usual assumption of orientability of the closed geodesic (see [12]). The Morse index theorem is given in terms of a symplectic invariant of the geodesic, called the Maslov index. The rate of growth of this index under iteration, which is one of the main points in Gromoll and Meyer paper, is studied in [8], where the authors prove that the index of an $n$ th iterate is either bounded by a universal constant, or it grows at least linearly in $n$ as $n \rightarrow \infty$.

As to the nullity of an iterate, the result is totally analogous to the Riemannian case using the linearized Poincaré map. This setup paves the path to an application of infinite dimensional equivariant Morse theory, in the same spirit as Gromoll and Meyer's celebrated result, that gives the existence of infinitely many critical points for the functional $\left.E\right|_{\tilde{\mathcal{N}}}$.

We will now state precisely the main result in [3]. Let $(M, g)$ be a globally hyperbolic stationary Lorentzian manifold, and let us assume that $M$ admits a complete timelike Killing vector field $\mathcal{Y}$. Denote by $\mathcal{F}_{t}, t \in \mathbb{R}$, the flow of $\mathcal{Y}$; clearly, if $\gamma$ is a (closed) geodesic in $M$, then also $\mathcal{F}_{t} \circ \gamma$ is a (closed) geodesic for all $t \in \mathbb{R}$.

In order to state the result, we need to give an appropriate notion of geometric equivalence of closed geodesics.

Definition. Given closed geodesics $\gamma_{i}:\left[a_{i}, b_{i}\right] \rightarrow M, i=1,2$, in a stationary Lorentzian manifold $(M, g)$, we will say that they are geometrically distinct, if there exists no $t \in \mathbb{R}$ such that the sets $\mathcal{F}_{t} \circ \gamma_{1}\left(\left[a_{1}, b_{1}\right]\right)$ and $\gamma_{2}\left(\left[a_{2}, b_{2}\right]\right)$ coincide.

The main result of [3] is the following:

Theorem. Let $(M, g)$ be a simply connected globally hyperbolic stationary Lorentzian manifold having a complete timelike Killing vector field, and having a compact Cauchy surface. Assume that the free loop space $\Lambda M$ has unbounded Betti numbers with respect to some coefficient field. Then, there are infinitely many geometrically distinct non trivial (i.e., non constant) closed geodesics in $M$.

Note that, by causality, every closed geodesic in $(M, g)$ is spacelike. It should be observed here that, although the notion of geometric equivalence given above depends on the choice of a complete timelike Killing vector field, the property of existence of infinitely many geometrically distinct closed geodesics is intrinsic to $(M, g)$. It is also interesting to observe that the statement of the Theorem admits a generalization to a class of non simply connected manifolds. Namely, the assumptions that $M$ is simply connected and that the Betti numbers of $\Lambda M$ form an unbounded sequence can be replaced by the single assumption that $\lim \sup _{k \rightarrow \infty} \beta_{k}(\Lambda M)=+\infty$. 
Examples of non simply connected spaces $M$ satisfying $\beta_{k}(\Lambda M ; \mathbb{K})=$ $+\infty$ for some small value of $k$ but for which $\beta_{k}(\Lambda M ; \mathbb{K})$ is bounded for $k$ large can be obtained as follows. Consider a standard stationary Lorentzian manifold $M=S \times \mathbb{R}$, where $S$ is a compact connected manifold whose universal cover is contractible. The free loop space $\Lambda M$ of $M$ is homotopically equivalent to the free loop space $\Lambda S$ of $S$. Given $p \in S$, denote by $\Omega_{p} S$ the loop space of $S$ based at $p$; the map $\Lambda S \ni \gamma \mapsto \gamma(0) \in S$ is a fibration, whose fiber at $p$ is $\Omega_{p} S$. The space $\Omega_{p} S$ has infinitely many connected component $\left(\pi_{1}(S)\right.$ must be infinite), each of which is contractible, by the assumption on the universal cover of $S$. It follows that each connected component of $\Lambda S$ is homotopically equivalent to $S$, and therefore, given any coefficient field $\mathbb{K}, \beta_{k}(\Lambda S, \mathbb{K})=+\infty$ for some $k \in\{0, \ldots, \operatorname{dim}(S)\}$, while $\beta_{k}(\Lambda S ; \mathbb{K})=0$ for all $k>\operatorname{dim}(S)$.

\section{References}

[1] A. Abbondandolo, P. Majer, A Morse complex for infinite dimensional manifolds. I, Adv. Math. 197 (2005), no. 2, 321-410

[2] J. K. Beem, P. E. Ehrlich, And K. L. Easley, Global Lorentzian geometry, vol. 202 of Monographs and Textbooks in Pure and Applied Mathematics, Marcel Dekker Inc., New York, second ed., 1996.

[3] L. Biliotti, F. Mercuri, and P. Piccione, On a gromoll-meyer type theorem in globally hyperbolic stationary spacetimes, Preprint, arXiv : math.DG/0701654, (2007).

[4] Bott, R. : Non-degenerate critical manifolds, Ann. of Math., 60 248-261 (1954)

[5] Bott, R. : On the iteration of closed geodesics and the Sturm intersection theory, Comm. Pure Appl. Math, 9 171-206 (1956)

[6] Gromoll, D. Mayer, W. On differentiable functions with isolated critical points, Topology 8, 361-369 (1969).

[7] Gromoll, D. Mayer, W. Periodic geodesics on compact Riemannian manifolds, J. Diff. Geo. bf 3 493-510 (1969).

[8] M. A. Javaloyes, P. Piccione, Iteration of closed geodesics in stationary Lorentzian manifolds, preprint 2207, to appear in Math. Z.

[9] Katok, A. B. : Ergotic perturbations of degenerate integrable Hamiltonian systems Math. USSR, Izvestija 7, 535-571 (1973).

[10] Klingenberg, W. Lectures on closed geodesics, Grundleheren der Mathematiscen Wissenschaften, 230 Springer-Verlag, Berlin, Heidelberg, New York, (1978).

[11] Klingenberg, W. Takens, , Generic properties of geodesic flows, Math. Ann. 197 (1972), pp. 323-334.

[12] R. C. Nostre Marques, P. Piccione, D. V. Tausk, On the Morse and the Maslov index for periodic geodesics of arbitrary causal character, Differential geometry and its applications (Opava, 2001), 343-358, Math. Publ. 3, Silesian Univ. Opava, Opava, 2001.

[13] Matthias, H. H. : Zwei Varallgemeineurungen eines Satzes von Gromoll-Meyer Bonner Mathematische Schriften 126 (1980).

[14] Mercuri, F. : The critical points theory for the closed geodesics problem Math. Z., 156, 231-245 (1977). 
[15] Mercuri, F. Palmieri, G. : Morse theory with low differentiability Boll. U.M.I., 7 1-B, 621-631 (1987).

[16] Milnor, J. W. : Morse Theory. Ann. of Math. Studies 51, Princeton Unuversity Press, 1963.

[17] Moura, A. A. Souza, F. M. : A Morse lemma for degenerate critical points with low differentiability. Abstr. Appl. Anal. 5 (2000), n.2, 113-118.

[18] O'Neill, B. Semi-Riemannian geometry, vol. 103 of Pure and Applied Mathematics, Academic Press Inc. Harcourt Brace Jovanovich Publishers, New York, 1983.

[19] Palais, R. : Morse theory on Hilbert manifolds, Topology, 2, 299-340 (1963).

[20] Palais, R. : Liusternik-Schnirelaman theory on Banach manifolds, Topology, 5, 115-132 (1966).

[21] Palais, R. Terng, C. : Critical point theory and submanifold geometry, Lecture notes in Mathematics 1353, Springer-Verlag, Berlin, Heidelberg, New York, (19??).

[22] Souza, F. M. : Teoria de Morse para o problema das geodésicas fechadas em variedades de Finsler, Doctoral dissertation, Universidade Estadual de Campinas, Brazil, (1997).

[23] Vigué-Poirrier, M. Sullivan, D. :The homology theory for the closed geodesic problem, J. Diff. Geo. 11, 633-644 (1977).

[24] Ziller, W. : The geometry of Katok example, Ergotic Theory and Dyn. Syst., 3 135-157 (1983) 